\title{
Rapid Determination of Clonality by Detection of Two Closely-linked X Chromosome Exonic Polymorphisms Using Allele-specific PCR
}

\author{
Yunying Liu, ${ }^{\star}$ John Phelan, ${ }^{\star}$ Rodney C.P. Go, ${ }^{\star}$ Jaroslav F. Prchal, ${ }^{\ddagger}$ and Josef T. Prchal* \\ *Division of Hematology/Oncology, the Department of Epidemiology, School of Public Health, University of Alabama at Birmingham, \\ and Birmingham Department of Veteran’s Affairs Medical Center, Birmingham, Alabama, and ${ }^{\ddagger}$ cGill University, Montreal, Canada
}

\begin{abstract}
We reported two specific, reproducible, and quantitative clonality assays based on detection of exonic polymorphisms of the $X$ chromosome genes p55 and G6PD using rtPCR-LDR. These assays are inconvenient for screening purposes. This study sought to develop a simple, reproducible assay, practical for screening genomic DNA samples for p55/G6PD genotypes, rapid clonality determination, and to determine the linkage relationship between these closely related loci. The salient feature of ASPCR is the performance of two PCR rounds. The first generates template; the second, using one aliquot of first-round products in two reaction tubes, each containing one allele-specific primer, detects each allele. ASPCR and rtPCR-LDR produced identical p55/G6PD results in 91 normal female genomic DNAs, and in 12 clonal hematopoietic disorder cDNAs, confirming assay validity. 209 female and 207 male genomic DNA samples were analyzed for p55/G6PD genotype by ASPCR; 60\% of females were heterozygous at one or both loci. G6PD and p55 allelic frequencies were significantly different among African-American men and women, but were not significantly different among Caucasian men and women. These loci were in linkage equilibrium among African Americans, but not among Caucasians. ASPCR is a rapid, sensitive, and specific method for screening large numbers of genomic DNAs, and for rapid clonality determination. (J. Clin. Invest. 1997. 99:1984-1990.) Key words: allele specific PCR • clonality • gene frequency • G6PD • p55
\end{abstract}

\section{Introduction}

Clonality assays based on the Lyon/Beutler hypothesis of random $\mathrm{X}$ chromosomal inactivation $(1,2)$ have been instrumental in broadening knowledge about normal and abnormal hematopoiesis. The first clonality assays exploited electro-

Yunying Liu and John Phelan contributed equally to this manuscript, and should both be considered first authors.

A portion of these data was presented in preliminary form at the 36th annual convention of the American Society of Hematology.

Address correspondence to Josef T. Prchal, Division of Hematology/Oncology, 513 Tinsley Harrison Tower, UAB Station, University of Alabama at Birmingham, Birmingham, AL 35294. Phone: 205934-2721; FAX: 205-934-1910.

Received for publication 7 October 1996 and accepted in revised form 16 January 1997.

J. Clin. Invest.

(C) The American Society for Clinical Investigation, Inc.

0021-9738/97/04/1984/07 \$2.00

Volume 99, Number 8, April 1997, 1984-1990 phoretically distinguishable G6PD isoenzymes present in Africans (30\% of African women are heterozygous) (2). Secondgeneration clonality assays employ methylation-sensitive restriction endonucleases to detect methylation pattern differences between transcriptionally active versus inactive $\mathrm{X}$ chromosome linked polymorphic loci (i.e., PGK [3, 4], HPRT [5], m27 beta $[6,7]$, and HUMARA $[8,9])$. These assays are limited by racial specificity, lack of quantitation, and inapplicability to nonnucleated cells such as platelets and reticulocytes. We recently developed a clonality assay based on transcriptional polymorphisms of two ubiquitously expressed $\mathrm{X}$ chromosome genes; G6PD (C/T at 1311) and palmitoylated membrane protein, p55 (G/T at cDNA 358) $(10,11,12)$. The assay uses reverse transcription polymerase chain reaction followed by ligase detection reaction $(\mathrm{rtPCR}-\mathrm{LDR})^{1}(10,11,12)$ to distinguish the transcriptionally active $\mathrm{X}$ chromosome. The assay is sensitive, specific, reproducible, quantitative, and applicable to nonnucleated cells. The screening of populations for informative subjects using polymerase chain reaction-ligase detection reaction (PCR-LDR), however, is laborious, time-consuming, expensive, and entails extended exposure to radioactive material. To overcome these disadvantages, we have modified the previously reported techniques of allele-specific oligonucleotide hybridization (ASOH) (13), PCR-ASOH (14), and PCR amplification of specific allele (PASA) (15) into a simpler assay (allele-specific PCR) for genotype screening and clonality determination based on G6PD and p55 exonic polymorphisms (16). Allele-specific PCR (ASPCR) requires two rounds of PCR. The first round generates a high concentration of DNA template (from either genomic DNA or cDNA) containing the polymorphic region of interest. The second round, using the first round products as a template, distinguishes each polymorphic allele by using allele-specific primers. Using ASPCR, 209 human female genomic DNA samples of defined racial groups were analyzed to determine the degree of heterozygosity (and thus informativeness for clonality studies) at these closely apposed X chromosome exonic polymorphic sites. 207 human male genomic DNA samples of defined racial groups were analyzed for G6PD and p55 haplotype determination, calculation of allelic frequencies, and determination of linkage equilibrium/disequilibrium. Linkage studies were conducted because multiple crossovers were noted, despite the observation that these two loci are proximate on the $\mathrm{X}$ chromosome. We have determined the respective allelic frequencies for both loci, and have conducted linkage analysis. In addition, we demonstrate that clonality can be determined rapidly, qualitatively, and without using radioactive material by employing rtPCR followed by ASPCR.

1. Abbreviations used in this paper: ASOH, allele-specific oligonucleotide hybridization; ASPCR, allele-specific PCR; LDR, ligase detection reaction; PASA, PCR amplification of a specific allele. 


\section{Methods}

Subjects. To examine large numbers of individuals for the polymorphic genotypes, peripheral blood samples from unselected individuals of three defined racial groups: Caucasians, African-American, and Asians (Chinese and Vietnamese) were used as a source of DNA.

Isolation of myeloid cells. Peripheral blood samples anticoagulated with EDTA were used. Granulocytes, mononuclear cells, platelets, and reticulocytes were separated by differential centrifugation, isopyknic density gradient separation, and dextran sedimentation using standard protocols (17).

DNA isolation and preparation. Genomic DNA samples were prepared from $5-10 \mathrm{ml}$ of peripheral blood. Red blood cells were lysed utilizing a $\mathrm{NH}_{4} \mathrm{HCO}_{3} / \mathrm{NH}_{4} \mathrm{Cl}$ buffer system. This was followed by overnight incubation at $37^{\circ} \mathrm{C}$ with nuclear membrane lysis buffer, $1 \%$ SDS, and proteinase $\mathrm{K}(1 \mathrm{U} / \mathrm{ml}$; Applied Biosystems Inc., Foster City, CA). Impurities were precipitated out with saturated $\mathrm{NaCl}$ (18), and DNA was extracted with $2 \mathrm{vol}$ of $100 \%$ ethanol followed by $70 \%$ ethanol. After air-drying, the DNA pellet was dissolved in 200-400 $\mu \mathrm{l}$ of TE buffer or distilled/deionized water, and stored at $4^{\circ} \mathrm{C}$.

Preparation of $m R N A$. mRNA was prepared by acid guanidium thiocyanate-phenol/chloroform extraction and ethanol precipitation from the isolated myeloid cells (19). RNA samples were dissolved in water treated with DEPC, and were stored at $-80^{\circ} \mathrm{C}$ until analysis.

Reverse transcription of $m R N A$. The aliquots of mRNA purified from separated myeloid cells were transcribed to first-strand cDNA using SuperScript RT (GIBCO BRL, Gaithersburg, MD) with random hexanucleotide primers (Pharmacia Biotech, Uppsala, Sweden).

Allele-specific PCR. ASPCR consists of two rounds of PCR (see Fig. 1). In the first round, high concentration and purity products containing the region of interest are generated. $1-10 \mu$ l of genomic DNA, or $5 \mu \mathrm{l}$ of cDNA is added to a $50-\mu \mathrm{l}$ reaction volume composed of
PCR buffer, $2 \mathrm{mM} \mathrm{MgCl}, 1$ unit of Taq polymerase (GIBCO BRL), $0.2 \mathrm{mM}$ dNTP, $25 \mathrm{pmol}$ of forward primer $(6 \mathrm{~J}-\mathrm{p} 55$ or $7 \mathrm{~g}-\mathrm{G} 6 \mathrm{PD})$, and 25 pmol of reverse primer (8gR-p55 or 9gR-G6PD). 35 cycles are carried out in a GeneAmp PCR System 9600 (Perkin Elmer, Emeryville, CA). Each cycle consisted of $40 \mathrm{~s}$ at $94^{\circ} \mathrm{C}$ for denaturation, $1 \mathrm{~min}$ at $62^{\circ} \mathrm{C}$ for annealing, and $1 \mathrm{~min}$ at $72^{\circ} \mathrm{C}$ for elongation. The second round was carried out in two reaction tubes. Each tube contained a $50-\mu$ reaction volume composed of PCR buffer, $1.5 \mathrm{mM}$ of $\mathrm{MgCl}_{2}$, $1 \mathrm{U}$ of Taq polymerase (GIBCO BRL), $0.2 \mathrm{mM}$ dNTP, and $10 \mathrm{pmol}$ of allele-specific primer as forward primer (e.g., $1 \mathrm{gT}$ or $3 \mathrm{gG}$ for $\mathrm{p} 55 /$ $3 \mathrm{C}$, or $4 \mathrm{~T}$ for G6PD), and the same reverse primer as that used in the first round of PCR (8gR for $\mathrm{p} 55$ or $9 \mathrm{gR}$ for $\mathrm{G} 6 \mathrm{PD}$ ). $5 \mu$ l of first-round products was used as a template for the second round. The second round consisted of five cycles performed in the same GeneAmp PCR system $\left(30 \mathrm{~s}\right.$ at $94^{\circ} \mathrm{C}$ for denaturing; $1 \mathrm{~min}$ at $64^{\circ} \mathrm{C}$ for annealing, and 1 min at $72^{\circ} \mathrm{C}$ for elongation). $15 \mu \mathrm{l}$ of products (from each secondround reaction tube) were then analyzed directly on $1 \%$ agarose gel with $0.5 \mu \mathrm{g} / \mathrm{ml}$ ethidium bromide. The first-round products were always visualized under UV light as the same high molecular weight band on both first- and second-round agarose gels, and thus served as an internal control. The sequences of the oligonucleotide primers employed in the ASPCR assay are depicted in Table I.

For rapid determination of clonality, the template for the first round of PCR was cDNA synthesized from total RNA by reverse transcription. Second-round PCR was conducted in an identical fashion as with genomic DNA.

Ligase detection reaction $(L D R)$. The LDR assays of p55 and G6PD were performed using PCR products generated from genomic DNA or cDNA templates as previously reported (10-12). The 10- $\mu \mathrm{l}$ reaction volume consisted of the following: (a) $10 \mathrm{ng}$ of template, $(b)$ $20 \mathrm{U}$ of cloned thermostable ligase (20) (a kind gift of Dr. Barany), (c) 5 pmol of each allele-specific oligonucleotide (as $5^{\prime}$ oligonucleotides),

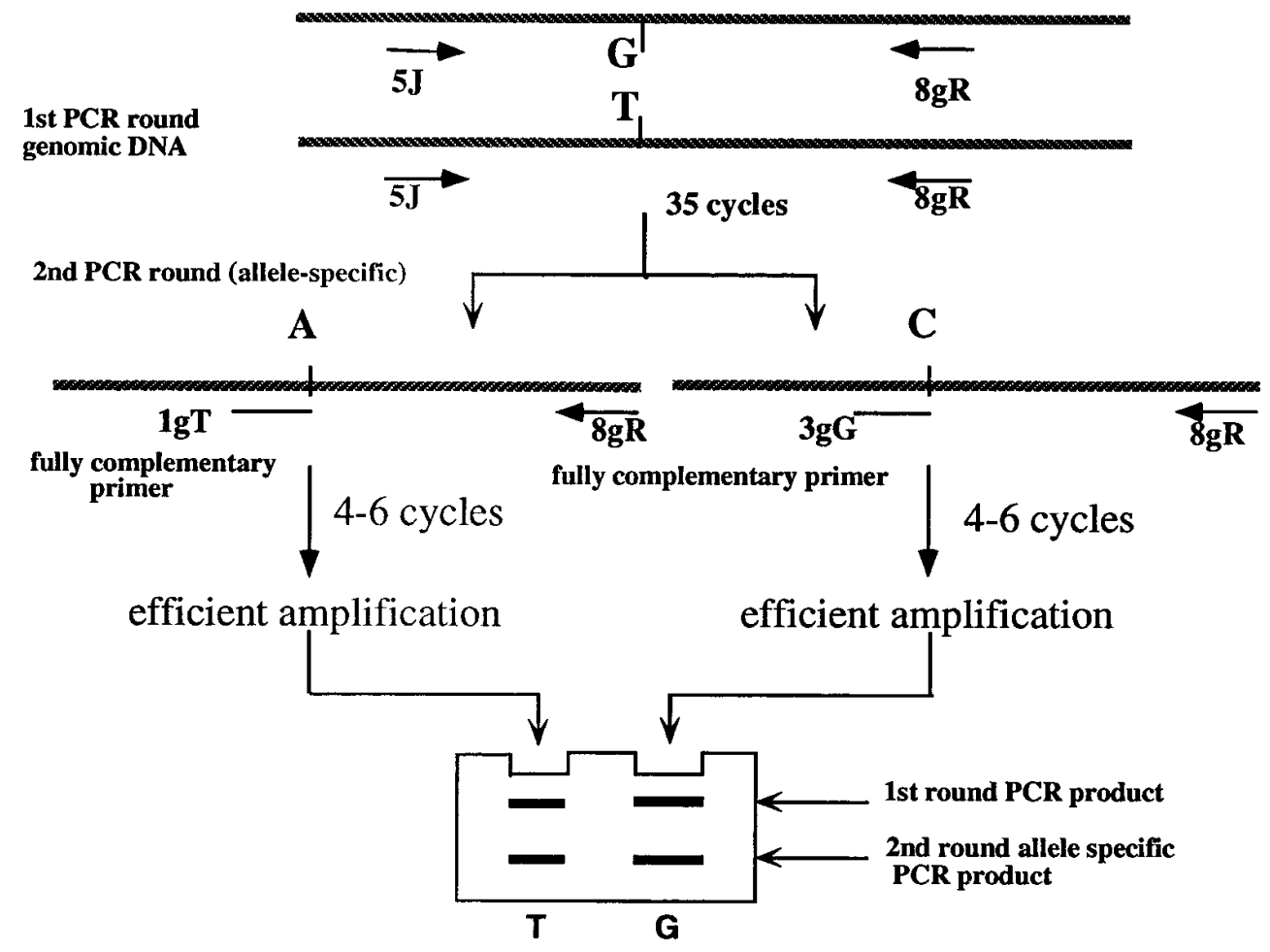

heterozygous for $\mathbf{T \& G}$ alleles
Figure 1. Outline of allele-specific PCR (ASPCR) using detection of p55 X chromosome exonic polymorphism $(\mathrm{G} / \mathrm{T}$ at cDNA 358) as an example: detection of $\mathrm{G}$ and $\mathrm{T}$ alleles in a heterozygous individual. Firstround PCR products serve as an internal control. Well-designated $\mathrm{G}$ has $\mathrm{G}$-specific allele primers. Well-designated $\mathrm{T}$ has T-specific allele primers. Genomic DNA from homozygote individuals will generate secondround allele-specific products in only one well. Genomic DNA from heterozygous individuals will generate second-round allele-specific products in both wells. Clonal samples (using cDNA from heterozygous patients) will show second-round allele-specific products in only one well. Nonclonal samples (using cDNA from heterozygous patients) will show secondround allele-specific products in both wells. The same principles apply to procedures used for detection of G6PD X chromosome exonic polymorphism (C/T 1311) by this assay. 
Table I. Primer Sequences Used in ASPCR for p55 and G6PD

\begin{tabular}{|c|c|c|c|c|c|}
\hline Gene & Primer & Length & Sequence $5^{\prime}$ to $3^{\prime}$ & Temp. & Paired with \\
\hline & & $b p$ & & ${ }^{\circ} \mathrm{C}$ & \\
\hline \multirow[t]{7}{*}{ p55 } & 6J \#/@ & 25 & TCAAGGGACAGGAGGTGCGGAAAGT & 57 & $8 g R$ \\
\hline & $9 \mathbf{J} @$ & 25 & CAGAGGCTGTATCGCATCCATTGAA & 53.2 & $8 \mathrm{gR}$ \\
\hline & 8gR\#/@ & 25 & TTTGGTTTCTTTCATCGTCTTCTGC & 54 & $6 \mathrm{~J} / 9 \mathrm{~J}$ \\
\hline & 1gT\# & 24 & TCGCCTTTATTTCAGGGAATCACT & 70.5 & $8 \mathrm{gR}$ \\
\hline & $3 \mathrm{gG \#}$ & 26 & TATCGCCTTTATTTCAGGGAATCACG & 71 & $8 \mathrm{gR}$ \\
\hline & 1T@ & 21 & TCAGAGCCCATGGGAATCACT & 74 & $8 \mathrm{gR}$ \\
\hline & 2G@ & 23 & TCTCAGAGCCCATGGGAATCAC & 78 & $8 \mathrm{gR}$ \\
\hline \multirow[t]{8}{*}{ G6PD } & $7 \mathrm{~g} \#$ & 21 & TTTGCAGCCGTCGTCCTCTAT & 47.6 & $9 \mathrm{gR}$ \\
\hline & $9 \mathrm{gR} \#$ & 21 & TGCAGCAGTGGGGTGAAAATA & 46.6 & $7 \mathrm{~g}$ \\
\hline & 3C\#/@ & 23 & CTGTGAAGCTCCCTGACGCCTAC & 55.7 & $9 \mathrm{gR}$ \\
\hline & 4T \#/@ & 25 & CTTTGTGAAGCTCCCTGACGCCTAT & 55.7 & $9 \mathrm{gR}$ \\
\hline & $1 @$ & 20 & TGATGACCAAGAAGCCGGGC & 60.8 & $2 \mathrm{R}$ \\
\hline & 2R@ & 20 & TGGGCTTGGGCTTCTCCAGC & 62.3 & 1 \\
\hline & 3@ & 20 & CTTCAACCCCGAGGAGTCGG & 59.5 & $4 \mathrm{R}$ \\
\hline & 4R@ & 20 & TCTGGTGCAGCAGTGGGGTG & 60.5 & 3 \\
\hline
\end{tabular}

For the second PCR round, in p55 ASPCR assay, different forward primers for genomic DNA and cDNA analysis are required because of the nearness of the polymorphic site to the intron/exon boundary (\# denotes allele specific primers for genomic DNA, @ for cDNA). Forward primers for genomic DNA and cDNA in G6PD ASPCR are the same, because the polymorphic site is located well within exon 11.

and $(d)$ the common downstream ligation oligonucleotide partner $\left(5^{\prime}\right.$ end-labeled with ${ }^{32} \mathrm{P}$ by T4 kinase). Five cycles $\left(1 \mathrm{~min}\right.$ at $94^{\circ} \mathrm{C}, 4 \mathrm{~min}$ at $64^{\circ} \mathrm{C}$ ) were performed using a Thermocycler 480 (Perkin Elmer Applied Biosystems, Foster City, CA). $4 \mu$ of the resulting reaction products plus $9 \mu \mathrm{l}$ of formamide stop buffer were then loaded onto a $15 \%$ polyacrylamide gel with $7 \mathrm{M}$ urea. After $2 \mathrm{~h}$ of electrophoresis at $60 \mathrm{~W}$, products were visible on the autoradiogram (see Fig. 2).

Calculations of gene allele frequencies and linkage disequilibrium. Maximum likelihood methods (21) were used to calculate the gene frequencies of the different alleles given the genotypic distributions in each racial group. The $z$ test for difference in binomial proportions (22) was used to identify any significant gene frequency differences among men and women of specified racial groups (race and gender). A significant difference among the genders indicated that the gene frequencies at these $\mathrm{X}$-linked loci were not in equilibrium. To test for significant deviations from linkage equilibrium $(\mathrm{D}=0)$, a Chi-square test (23-25) was applied to the male haplotype data.

\section{Results}

Specificity of ASPCR. Identical genotypes were obtained in all 91 female genomic DNA samples analyzed simultaneously with ASPCR and PCR-LDR p55 or G6PD assays. In Fig. 2, parallel data obtained by two methods of three individuals tested in p55 assay and of another three individuals tested in
G6PD assay are depicted, demonstrating that ASPCR reliably determines both G6PD and p55 exonic X chromosome polymorphic genotypes.

Determination of clonality by rtPCR-ASPCR. 12 heterozygous females known to have clonal hematopoietic disorders as defined by rtPCR-LDR assay, had cDNA of their clonal tissues reevaluated using the rtPCR-ASPCR assay. In all 12, the same clone-defining allele previously identified by rtPCRLDR was similarly identified by rtPCR-ASPCR. This demonstrates that rtPCR-ASPCR is suitable as a qualitative clonality assay. ASPCR analysis (p55 assay) of peripheral blood platelets of patients 1 and 2 as well as that of a subject with a nonclonal hematological disorder (patient 3 ) is shown in Fig. 3. Similar data were obtained for G6PD (data not shown). Genomic DNA analysis, documenting p55 heterozygosity is shown in $A$ and cDNA analysis identifying p55 clonality is shown in $B$.

Frequency of heterozygosity (Table II). Among the 209 human female genomic DNA samples analyzed, $48 \%$ were heterozygous in the p55 locus, and $27 \%$ were heterozygous in the G6PD locus. When both loci were considered together, $43 \%$ of African-Americans, $50 \%$ of Asians, and $44 \%$ of Caucasians were heterozygous at either locus. Moreover, $18 \%$ of AfricanAmerican, $9 \%$ of Asian, and 15\% of Caucasian females were

Table II. Exonic Polymorphism Genotypes and Genotype Frequencies in African-American, Asian, and Caucasian Women

\begin{tabular}{|c|c|c|c|c|c|c|c|c|}
\hline & \multicolumn{4}{|c|}{ p55 } & \multicolumn{4}{|c|}{ G6PD } \\
\hline & $n$ & Homozygous-GG (p) & Homozygous-TT (q) & Heterozygous-G/T (pq) & $n$ & Homozygous-CC (p) & Homozygous-TT (q) & Heterozygous-C/T (pq) \\
\hline African-American & 49 & $18(0.37)$ & $10(0.20)$ & $21(0.43)$ & 49 & $28(0.57)$ & $3(0.06)$ & $18(0.37)$ \\
\hline Caucasian & 126 & $19(0.15)$ & $41(0.33)$ & $66(0.52)$ & 126 & $95(0.75)$ & $2(0.02)$ & $29(0.23)$ \\
\hline Asian & 34 & $4(0.12)$ & $16(0.47)$ & $14(0.41)$ & 34 & $24(0.71)$ & $1(0.03)$ & $9(0.26)$ \\
\hline Total & 209 & $41(0.20)$ & $67(0.32)$ & $101(0.48)$ & 209 & $147(0.70)$ & $6(0.03)$ & $56(0.27)$ \\
\hline
\end{tabular}


C.
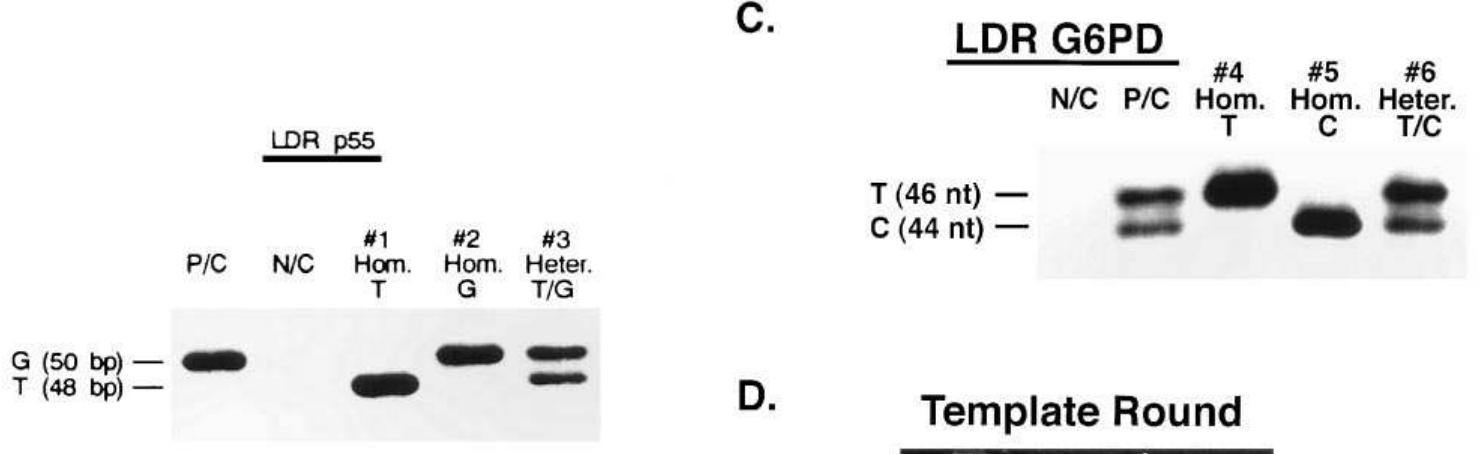

D.

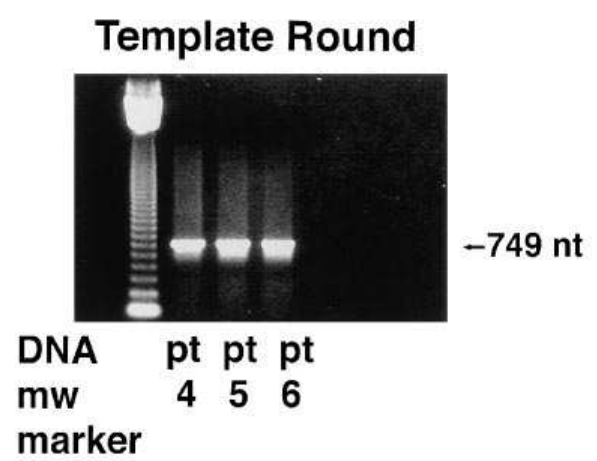

\section{Allele Specific Round}

B.

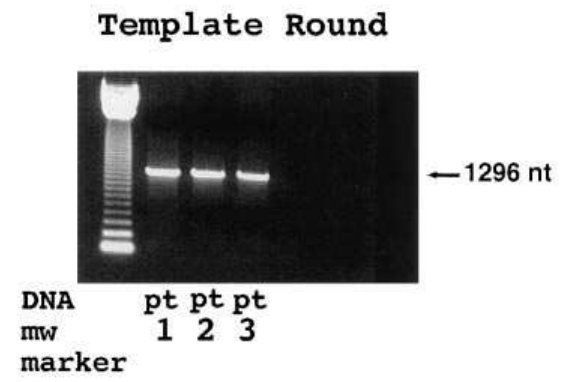

Allele Specific Round

T $\mathbf{G} \quad \mathbf{T}$ G $\quad$ T $\mathbf{G}$
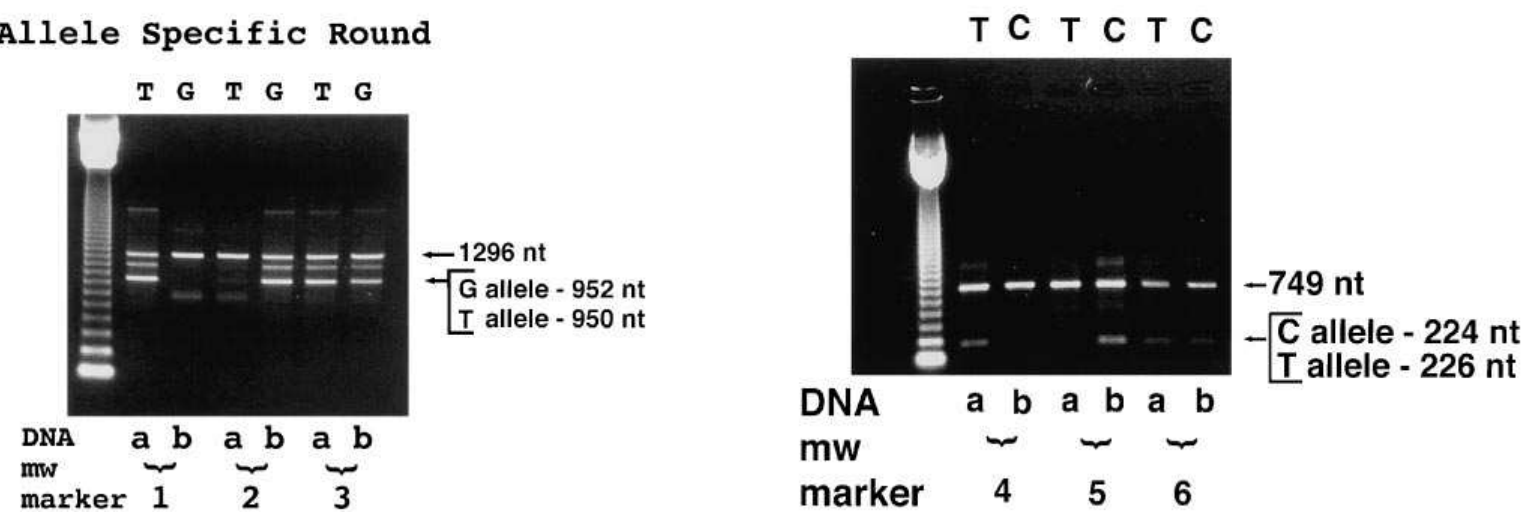

Figure 2. Specificity of ASPCR. Representative p55 exonic polymorphism genotype analysis of three human female genomic DNA samples analyzed by rtPCR-LDR $(A)$ and ASPCR $(B)$. $(A)$ Representative PAGE/autoradiogram of rtPCR-LDR for p55 exonic polymorphism genotype analysis of three human female genomic DNA samples. G designates a 50-bp ligation product consisting of a $\mathrm{G}$ allele-specific oligonucleotide ligated to a radiolabeled common downstream oligonucleotide. T designates a 48-bp ligation product consisting of a T allele-specific oligonucleotide ligated to a radiolabeled common downstream oligonucleotide. Lane $P / C$ represents a positive control using genomic DNA from a known $\mathrm{G}$ allele homozygote. Lane N/C represents a negative control wherein the genomic DNA template was substituted with water before the LDR step. Lane 1 represents analysis of individual homozygous for T allele; lane 2 represents analysis of individual homozygous for $\mathrm{G}$ allele; and lane 3 represents individual heterozygous for $\mathrm{G}$ and $\mathrm{T}$ alleles. $(B)$ Representative agarose gel electrophoreses of ASPCR for p55 exonic polymorphism genotype analysis of three human female genomic DNA samples (same individuals as in $A$ ). Template round represents 35 -cycle amplification of that portion of the gene containing the polymorphism of interest ( $p 55$ gene in this instance). The high concentration/purity product from this round serves as a template for the next round (allele-specific round). In the second (or allele-specific) round, two aliquots from each template round reaction are subjected to six cycles of PCR amplification; an allele-specific forward primer (G-specific or T-specific for the p55 locus) is used for each reaction. Under these PCR conditions, only perfectly complementary primer-template pairs will undergo amplification efficiently enough to generate products visible by agarose gel electrophoresis. Each individual tested is thus represented by two gel lanes. The topmost bright band in each lane represents first-round generated template, and serves as an internal control. The presence of a second bright band demonstrates an allele-specific PCR product. Lane $1 a / b$ represents an individual homozygous for the p55 T allele. Lane $2 a / b$ represents an individual homozygous for the $\mathrm{G}$ allele. Lane $3 a / b$ represents an individual heterozygous for both the $\mathrm{G}$ and $\mathrm{T}$ alleles. The fainter band between the first and allele-specific round products represents heterodimer formation. $(C)$ Representative PAGE/autoradiogram of rtPCR-LDR for G6PD exonic polymorphism genotype analysis of three human female genomic DNA samples. C designates a 46-bp ligation product consisting of a $\mathrm{C}$ allele-specific oligonucleotide ligated to a radiolabeled common downstream oligonucleotide. T designates a 44-bp ligation product consisting of a T allele-specific oligonucleotide ligated to a radiolabeled common downstream oligonucleotide. Lane $P / C$ represents a positive control using genomic DNA from a known G allele homozygote. Lane $N / C$ represents a negative control wherein the genomic DNA template was substituted with water before the LDR step. Lane 1 represents analysis of individual homozygous for T allele; lane 2 represents analysis of individual homozygous for $\mathrm{C}$ allele; and lane 3 represents individual heterozygous for $\mathrm{C}$ and $\mathrm{T}$ alleles. $(D)$ Representative agarose gel electrophoreses of ASPCR for G6PD exonic polymorphism genotype analysis of three human female genomic DNA samples. Template round represents 35- 
A.

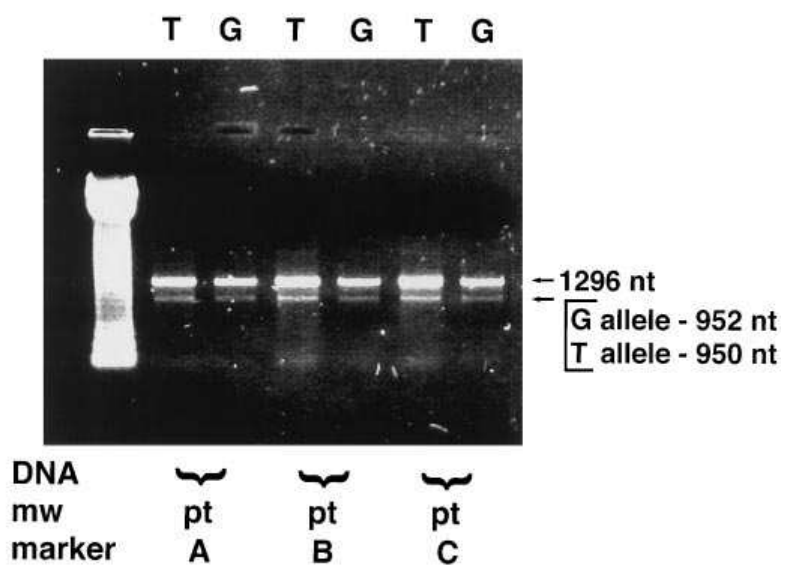

B.

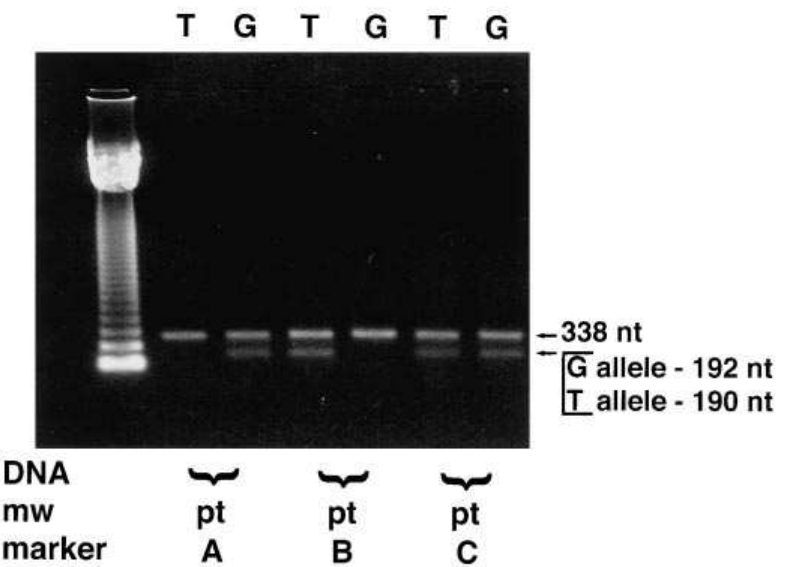

Figure 3. Determination of clonality by rtPCR-ASPCR. (A) Screening ASPCR (allele-specific round depicted) performed on genomic DNA isolated from the peripheral blood mononuclear cells of three females with hematological abnormalities. All are identified as heterozygous for the G/T exonic polymorphism at the p55 locus. Patient A was diagnosed with Fanconi anemia; patient B was diagnosed with essential thrombocythemia; and patient $\mathrm{C}$ was found not to have a primary hematological disorder. $(B)$ Total RNA was isolated from peripheral blood of patients $\mathrm{A}, \mathrm{B}$, and $\mathrm{C}$, subjected to reverse transcription (using p55 specific primers), and the resulting cDNAs were subjected to ASPCR. The allele-specific round is depicted here. Patients A and B, though genotypically heterozygous at the p55 locus, are here shown to transcribe only one allele, and thus demonstrate clonal hematopoiesis. Patient $\mathrm{C}$ demonstrates nonclonal hematopoiesis.
Table III. Exonic Polymorphism Allele Number and Allelic Frequencies in African-American and Caucasian Males and Females and Asian Females (Determined in Females by Chromosome Counting)

\begin{tabular}{|c|c|c|c|c|c|}
\hline \multirow[b]{2}{*}{ Allele } & \multicolumn{2}{|c|}{ African-Americans } & \multicolumn{2}{|c|}{ Caucasians } & \multirow{2}{*}{$\begin{array}{c}\text { Asians } \\
\text { Females }\end{array}$} \\
\hline & Females & Males & Females & Males & \\
\hline p55 & 98 & 105 & 252 & 102 & 68 \\
\hline G & $57(0.58)$ & $78(0.74)$ & $104(0.41)$ & $37(0.36)$ & $22(0.32)$ \\
\hline $\mathrm{T}$ & $41(0.42)$ & $27(0.26)$ & $148(0.59)$ & $65(0.64)$ & $46(0.68)$ \\
\hline G6PD & 98 & 105 & 252 & 102 & 68 \\
\hline $\mathrm{C}$ & $74(0.76)$ & $92(0.88)$ & $219(0.87)$ & $92(0.90)$ & $57(0.84)$ \\
\hline $\mathrm{T}$ & $24(0.24)$ & $13(0.12)$ & $33(0.13)$ & $10(0.10)$ & $11(0.16)$ \\
\hline
\end{tabular}

In 209 women and in 207 men, there are 615 alleles total.

heterozygous at each locus (doubly heterozygous). When combined, $60 \%$ of the human females analyzed were heterozygous at one or both loci, and thus were potentially informative for further clonality evaluation.

p55 and G6PD allelic frequencies. Genomic DNA samples from 209 human females of African-American, Asian, and Caucasian origin, and 207 human males of African-American and Caucasian origin (representing a total of $615 \mathrm{X}$ chromosomes) were analyzed by ASPCR. Allelic frequencies for both males' and females' p55 and G6PD exonic polymorphisms are depicted in Table III. The $z$ test for difference in binomial proportions was used to test for statistically significant allelic frequency differences between the genders in the African-American and Caucasian racial groups. The intergender p55 and G6PD allelic frequencies were significantly different in African-Americans $(P=0.016$ and $P=0.025)$, but not in Caucasians $(P=0.384$ and $P=0.435)$.

Linkage disequilibrium studies. The allelic frequencies of the p55 and G6PD haplotypes in male subjects of AfricanAmericans and of Caucasians are depicted in Table III $A$ and $B$. The data were subjected to linkage disequilibrium analysis, which demonstrated that the p55 and G6PD exonic loci were in linkage equilibrium among African-Americans $(\mathrm{D}=0.00625$, $\left.\chi^{2}=0.1977, P>0.05\right)$, but not among Caucasians $(\mathrm{D}=$ $\left.-0.04372, \chi^{2}=9.29, P<0.005\right)$.

\section{Discussion}

We have reported two novel clonality assays (rtPCR-LDR of the transcriptional products of the G6PD/p55 exonic polymorphisms) which reliably quantify active $\mathrm{X}$ chromosome transcrip-

Figure 2 legend (Continued)

cycle amplification of that portion of the gene containing the polymorphism of interest (G6PD gene in this instance). The high concentration/purity product from this round serves as a template for the next round (allele-specific round). In the second (or allele-specific) round, two aliquots from each template round reaction are subjected to six cycles of PCR amplification; an allele-specific forward primer (C-specific or T-specific for the G6PD locus) is used for each reaction. Under these PCR conditions, only perfectly complementary primer-template pairs will undergo amplification efficiently enough to generate products visible by agarose gel electrophoresis. Each individual tested is thus represented by two gel lanes. The topmost bright band in each lane represents first-round generated template, and serves as an internal control. The presence of a second bright band demonstrates an allele-specific PCR product. Lane $1 a / b$ represents an individual homozygous for the G6PD T allele. Lane $2 a / b$ represents an individual homozygous for the $\mathrm{C}$ allele. Lane $3 a / b$ represents an individual heterozygous for both the $\mathrm{C}$ and $\mathrm{T}$ alleles. 
tional products $(10,11)$. We have verified the reproducibility, sensitivity, and quantitative nature of rtPCR-LDR (10-12). Nonetheless, the rtPCR-LDR assays are cumbersome, labor intensive, time-consuming, and require working with radiolabeled oligonucleotides. The rtPCR-ASOH method, reported by Beutler's laboratory (26), though simpler to carry out, still requires blotting, radiolabeled oligonucleotide hybridization, and autoradiography. We believe that ASPCR, when utilized for genotype determination or as a qualitative clonality assay (as reported herein), may enjoy an advantage over ASOH by dispensing with the use of radioisotopes and time-consuming blotting, hybridization, and autoradiography steps. Furthermore, we demonstrate ASPCR specificity in that 91 genomic DNA samples analyzed concomitantly by ASPCR and PCRLDR produced identical genotype results. We did not conduct any direct comparison between ASPCR and ASOH for minor allele sensitivity of detection.

This ASPCR assay is a modification of the previously reported ASPCR (13), PCR-ASOH (14) and PCR amplification of specific alleles (PASA) (15). The utility of PASA for this type of application is limited because the mismatched pairing which occurs at the $3^{\prime}$ end of the primer-template duplex (i.e., $\mathrm{T}: \mathrm{C}, \mathrm{T}: \mathrm{G}, \mathrm{T}: \mathrm{T})$ is amplified by Taq polymerase as efficiently as the perfectly complementary primer-template duplexes. $\mathrm{Xu}$ and Hall (27) attempted to overcome this difficulty by manipulation of as many as 12 reaction variables (19). Their assay successfully analyzed the Escherichia coli genome; when we applied their assay to human genomic DNA, bands representing nonspecific products were always apparent on agarose gel electrophoresis. Reducing the number of cycles in the second round from $>20$ to 5, and assuring the presence of high template concentration eliminates the tendency of Taq polymerase to amplify primer-template mismatches, substantially decreasing the amount of nonspecific products while retaining allele-specific products. The entire ASPCR procedure can be completed in $6 \mathrm{~h}$; PCR-LDR can take as long as 2 or $3 \mathrm{~d}$. ASPCR specificity is not limited by the expected variation in concentration or quality of genomic DNA or cDNA. In addition, the same PCR conditions are employed in the analysis of p55 and G6PD, allowing for simultaneous determination of both genotypes. Unlike ASOH or PCR-ASOH, performance of ASPCR for genotype determination does not require extensive restriction endonuclease digestion of sample DNA, radiolabeled oligonucleotides, or time-consuming autoradiography.

The p55 and G6PD loci are $\sim 200 \mathrm{~kb}$ apart on the X chromosome (28). They are in linkage equilibrium among AfricanAmericans, but not among Caucasians. Linkage disequilibrium found in the Caucasian males may result from epistatic effects, genetic hitchhiking to a selected locus, or population subdivision (combining different ethnic groups with different allele frequencies). Which of these mechanisms is responsible for this phenomenon cannot be determined from these data alone.

The allelic frequencies are significantly different between the sexes in African-Americans, but not in Caucasians. The immediate reason for this fact is unclear. Moreover, there is no statistically significant difference among the relative allelic frequencies for either the p55 or G6PD locus among the human racial groups studied to date. This suggests that these polymorphisms were evolutionarily well-established before the geographic separation of humans into currently recognized racial groups. Interestingly, examination of Table III reveals a rela- tive paucity of the G6PD T allele in males, and of the T/T genotype in females among all racial groups analyzed.

The ASPCR methodology is suitable for automation and further simplification. The identification of other X chromosome polymorphic loci should increase the proportion of females suitable for research and clinical purposes such as rapid discrimination of malignant (clonal) from nonmalignant (polyclonal) tissue. The underlying principles of ASPCR can and have been already used (29) for rapid determination of mutations, genotypes, and polymorphisms for diverse clinical and nonclinical purposes.

\section{Acknowledgments}

This work was supported in part by the Office of Research and Development, Medical Research Service, Department of Veteran's Affairs, VAH Merit grant (J.T. Prchal), and United States Public Health Service grants 1R01 HL-51650 and HL-50077 (J.T. Prchal).

\section{References}

1. Lyon, M.F. 1988. The William Allan Memorial Award address: X-chromosome inactivation and the location and expression of X-linked genes. Am. J. Hum. Genet. 42:8-16.

2. Beutler, E., Z. Collins, and L.E. Irwin. 1967. Value of genetic variants of glucose- 6 phosphate dehydrogenase in tracing the origin of malignant tumors. N. Engl. J. Med. 276:389-391.

3. Keith, D.H., J. Singer-Sam, and A.D. Riggs. 1986. Active X chromosome DNA is unmethylated at eight CCGG sites clustered in a guanine-plus-cytosine-rich island at the $5^{\prime}$ end of the gene for phosphoglycerate kinase. Mol. Cell. Biol. 6:4122-4125.

4. Vogelstein, B., E.R. Fearon, and S.R. Hamilton. 1987. Clonal analysis using recombinant DNA probes from the X-chromosome. Cancer Res. 47:48064813.

5. Vogelstein, B., E.R. Fearon, S.R. Hamilton, and A.P. Feinberg. 1985 Use of restriction fragment length polymorphisms to determine the clonal origin of human tumors. Science (Wash. DC). 227:642-645.

6. Boyd, Y., and N.J. Fraser. 1990. Methylation patterns at the hypervariable X chromosome locus DXS255 (M27 beta): correlation with X-inactivation status. Genomics. 7:182-187.

7. Fey, M.F., S. Liechti-Gallati, and A. von Rohr. 1994. Clonality and X-inactivation patterns in hematopoietic cell populations detected by the highly informative M27 beta DNA probe. Blood. 83:931-938.

8. Allen, R.C., H.Y. Zoghbi, A.B. Moseley, H.M. Rosenblatt, and J.W. Belmont. 1992. Methylation of HpaII and HhaI sites near the polymorphic CAG repeat in the human androgen-receptor gene correlates with X-chromosome inactivation. Am. J. Hum. Genet. 51:1229-1239.

9. Busque, L., and D.G. Gilliland. 1993. Clonal evolution in acute myeloid leukemia. Blood. 82:337-342.

10. Prchal J.T., and Y.L. Guan. 1993. A novel clonality assay based on transcriptional analysis of the active X-chromosome. Stem Cells. 11(Suppl. 1):62-65.

11. Luhovy, M., Y. Liu, M. Belickova, J.F. Prchal, and J.T. Prchal. 1995. A novel clonality assay based on transcriptional polymorphism of X-chromosome gene p55. Biology of Blood and Marrow Transplantation. 1:81-87.

12. Prchal, J.T., J.F. Prchal, M. Belickova, S. Chen, Y. Guan, L. Gartland, and M.D. Cooper. 1996. Clonal stability of blood cell lineages indicated by X-chromosomal transcription polymorphism. J. Exp. Med. 183:748-760.

13. Wu, D.Y., L. Ugozzoli, B.J. Pal, and R.B. Wallace. 1989. Allele specific enzymatic amplification of beta globin genomic DNA for diagnosis of sickle cell anemia. Proc. Natl. Acad. Sci. USA. 86:2757-2760.

14. Saiki, R.K., T.L. Bugawan, G.T. Horn, K.B. Mullis, and H.A. Erlich. 1986. Analysis of enzymatically amplified Beta globin and HLA-DQ alpha DNA with allele-specific oligonucleotide probes. Nature (Lond.). 324:163-166.

15. Sommer, S.S., A.R. Groszbach, and C.D.K. Bottema. 1992. PCR amplification of specific alleles (PASA) is a general method for rapidly detecting known single base changes. Biotechniques. 12:82-87.

16. Liu, Y., M. Luhovy, and J.T. Prchal. 1994. A simple genotype screening assay for $\mathrm{X}$ chromosome transcriptional polymorphisms: allele specific PCR (ASPCR). Blood. 84(Suppl.):141.

17. Prchal, J.T., D.W. Throckmorton, A.J. Carroll III, E.W. Fuson, R.A. Gams, and J.F. Prchal. 1978. A common progenitor for human myeloid and lymphoid cells. Nature (Lond.). 274:590-591.

18. Miller, S.A., D.D. Dykes, and H.F. Polesky. 1988. A simple salting out procedure for extracting DNA from human nucleated cells. Nucleic Acids Res. 16:1215.

19. Chomczynski, P., and N. Sacchi. 1987. Single step method of RNA isola- 
tion by acid guanidium thiocyanate-phenol-chloroform extraction. Anal. Biochem. 162:156-159.

20. Barany, F. 1991. Genetic disease detection and DNA amplification using cloned thermostable ligase. Proc. Natl. Acad. Sci. USA. 88:189-193.

21. Li, C.C. 1976. First Course in Population Genetics. Boxwood Press, Pacific Grove, CA. pp. 2-15.

22. Snedecor, G.W., and W.G. Cochran. 1967. Statistical Methods. 6th ed. The Iowa State University Press, Ames, IA. pp. 215-219.

23. Bennett, J.H., and C.R. Oertel. The approach to random association of genotypes with random mating. J. Theoret. Biol. 9:67-76.

24. Hill, W.G. Estimation of linkage disequilibrium in randomly mating populations. Heredity. 33:229-239.
25. Hedrick, P.W. 1974. Genetics of Populations. Bartlett and Jones Publishers, Inc. Portola Valley, CA. pp. 382-383.

26. Curnutte, J.T., P.J. Hopkins, W. Kuhl, and E. Beutler. 1992. Studying X inactivation. Lancet. 339:749.

27. Xu, L., and B.G. Hall. 1994. SASA: A simplified, reliable method for allele-specific amplification of polymorphic sites. Biotechniques. 16:44-45.

28. Metzenger, A.B., and J. Gitschier. 1992. The gene coding the palmitoylated erythrocyte membrane protein p55 originates at the $\mathrm{CpG}$ island $3^{\prime}$ to the Factor VIII gene. Hum. Mol. Genet. 1:97-101.

29. Jenkins, M.M., and J.T. Prchal. 1996. A novel mutation found in the $3^{\prime}$ domain of NADH-cytochrome B5 reductase in an African-American family with type I congenital methemoglobinemia. Blood. 87:2993-2999. 